\title{
O Impacto da Relação Mãe-Filha no Desenvolvimento da Autoestima e nos Transtornos Alimentares ${ }^{1}$
}

\author{
Daniela Sopezki \\ Cícero E. Vaz \\ Pontifícia Universidade Católica do Rio Grande do Sul
}

\begin{abstract}
RESUMO
Nesse artigo dois fatores de risco para os transtornos alimentares são analisados, bem como sua interrelação contribuinte no desenvolvimento e manutenção da anorexia nervosa e da bulimia nervosa, em mulheres: a relação mãe-filha e a autoestima. Entre as necessidades humanas está a de estima, ou seja, a necessidade de autoestima e estima por parte dos outros. As mães tendem a vivenciar suas filhas mulheres como menos separadas delas, devido a componentes narcisistas que prevalecem nesta dupla como identificação e simbiose. No caso das mulheres com transtornos alimentares algo se inverteu no processo de interação entre mãe-filha prejudicando o vínculo entre elas. A formação da adequada autoestima depende profundamente do olhar amoroso de apreciação por uma pessoa significativa, a mãe, porque nunca é com seus próprios olhos que a criança se vê, mas sempre com os olhos do outro. $\mathrm{O}$ ver-se numa identificação com esse olhar dirigido para si constitui o narcisismo, a sua própria autoestima e dependendo do tipo de apego existente entre essa dupla, a autoestima da filha terá nuances diferenciadas.
\end{abstract}

Palavras-chave: transtornos alimentares; vínculo mãe-filha; autoestima.

\section{ABSTRACT \\ The Impact of the Mother and Daughter Relationship in the Development of Self-Esteem and Eating Disorders}

In this paper, two risk factors for eating disorders are analyzed, as well as their inter-relation to the development and maintenance of anorexia nervosa and bulimia nervosa among women specifically focusing on the mother-daughter relationship and self-esteem. A basic human need is one of esteem, in particular, the need for self-esteem and esteem from others. Mothers tended to treat their daughters as less separate from them, due to narcissistic components that prevail among them as identification and symbiosis. Among women with eating disorders, something has affected the mother-daughter interaction process weakening the bond between them. Since it is never through one's own eyes that children see themselves but always through the eyes of others, the proper development of self-esteem depends deeply on loving regard of a significant person, in this case, the child's mother. The process of seeing one's self in identification with this look constitutes the formation of narcissism and one's own self-esteem. Depending on the type of mother-daughter attachment, the child's self-esteem will differ.

Keywords: eating disorders; mother-daughter relations; self-esteem.

Entre os principais transtornos alimentares estão a anorexia nervosa e a bulimia nervosa. Esses transtornos costumam afetar mulheres jovens (90\% dos casos) com curso crônico, variável e com alto grau de morbidade e mortalidade (Assumpção \& Cabral, 2002; Pinzon \& Nogueira, 2004). A anorexia nervosa, por exemplo, é difícil de ser tratada e tem maior média de mortalidade entre os transtornos psiquiátricos, cerca de $0,59 \%$ ao ano. Este valor é cerca de doze vezes maior que a mortalidade das mulheres jovens na população geral (Agras, 2001; American Psychiatric Association [APA], 2006). Atualmente, em função do falecimento da modelo Ana Carolina Reston, morta aos 21 anos em decorrência de anorexia (Segatto, Padilla \& Frutuoso, 2006), a documentação de óbitos desse transtorno está em evidência na mídia.

O termo anorexia, etimologicamente, deriva do grego "na", deficiência ou ausência de, e "orexis", 
apetite, também significando aversão à comida, enjôo do estômago ou inapetência. As primeiras referências a essa condição surgem com o termo fastidium em fontes latinas da época de Cícero (106-43 a.C.) e vários textos do século XVI. Já a denominação mais específica "anorexia nervosa" surgiu com William Gull e Laségue no século XIX. O termo anorexia nervosa, atualmente, não é utilizado em seu sentido etiológico, visto que tais pacientes não apresentam real perda de apetite até estágios mais avançados da doença, mas sim uma recusa alimentar deliberada, com intuito de emagrecer ou por medo de engordar (Cordás \& Claudino, 2002).

De acordo com a American Psychiatric Association (2002), os critérios diagnósticos da anorexia nervosa são: recusa a manter o peso corporal em um nível igual ou acima do mínimo normal adequado à idade $\mathrm{e}$ à altura; medo intenso de ganhar peso ou de se tornar gorda, mesmo com o peso abaixo do normal; perturbação no modo de vivenciar o peso, ou a forma do corpo e a ausência de pelo menos três ciclos menstruais consecutivos.

Já o termo bulimia nervosa foi denominado por Russell (1979) e vem da união dos termos gregos boul (boi) ou bou (grande quantidade) com lemos (fome), ou seja, uma fome muito intensa ou suficiente para devorar um boi. É interessante lembrar que o comportamento de forçar o vômito é muito antigo e pode ser encontrado precocemente na história de diferentes povos da Antiguidade, que faziam uso da prática de vômitos, para prevenir ou curar doenças, poder aliviar-se de banquetes, entre outros (Cordás, 2004).

Os critérios diagnósticos para a bulimia nervosa são: episódios recorrentes de compulsão alimentar periódica; comportamento compensatório, inadequado e recorrente, com o fim de prevenir o aumento de peso, como autoindução de vômito, uso indevido de laxantes, diuréticos, jejuns ou exercícios excessivos; a compulsão e a compensação inadequadas ocorrem em média, pelo menos duas vezes por semana, por três meses; a autoavaliação é indevidamente influenciada pela forma e peso corporais e o distúrbio não ocorre exclusivamente durante episódios de anorexia nervosa (APA, 2002).

Uma série de interações multifatoriais (genéticas, socioculturais, vulnerabilidades biológicas e psicológicas) de componentes de risco e outros eventos precipitantes são responsáveis pelo surgimento dos transtornos alimentares (Morgan, Vecchiatti \& Negrão, 2002). Nesse artigo dois fatores de risco são analisa- dos, bem como sua inter-relação contribuinte no desenvolvimento e manutenção da anorexia nervosa e bulimia nervosa: a relação mãe-filha e a autoestima.

\section{O contexto sociocultural e o comportamento feminino}

É comum atribuir à cultura e à mídia uma grande parcela da influência nos comportamentos sociais. A cultura do culto ao corpo magro, atual padrão de beleza feminina, é perseguido desde as formas mais brandas às mais perigosas. Inchi e Meral (2006) orientam que há muita divulgação dos métodos utilizados pelas mulheres com transtornos alimentares, tais como maneiras de emagrecer, de purgar e de compensar, mais evidenciados do que os riscos que isto acarreta à saúde.

Predominantemente a mídia induz à idealização de imagens, promovendo a ideia de que o tamanho e a forma do corpo são flexíveis e facilmente modificados. As imagens parecem realísticas, graças ao processo de edição pelo qual passam e tal forma corporal divulgada pela mídia é biologicamente inatingível para a maioria das mulheres. Essa influência ocorre porque fatores sociais determinam, no adolescente, os valores acerca de si mesmo, exercendo forte influência na autoestima. Logo, a avaliação de si mesmo sempre acontece de acordo com certos critérios, que derivam de condições históricas de cada sociedade em particular (Rosenberg, 1973). Logo, o modelo contemporâneo de beleza, para as mulheres, é o da magreza, esse fator interfere no imaginário feminino.

A argumentação de Rosenberg (1973) a entender uma parcela da influência dos valores contemporâneos (corpo alto, magro, padrões de beleza rígidos), dado observado ao investigar que mulheres com poucas cognições positivas e muitas cognições negativas sobre si mesmas são particularmente mais vulneráveis às mensagens culturais sobre peso corporal e isto contribui para comportamentos alimentares anormais (Bardone \& Cass, 2006; Cameron \& Ferraro, 2004; Stein \& Corte, 2003).

Outros estudos (Monro \& Huon, 2006) têm tratado da influência que o contexto exerce, principalmente por meio da mídia, no comportamento alimentar feminino. Os autores submeteram mulheres à exposição de imagens contendo mensagens sobre corpo, estética e dietas, conseguindo identificar um impacto no seu comportamento alimentar, já Filippi (2004) ressalta que isso ocorre apenas quando há uma combinação de fatores predisponentes, já que esses transtornos possuem uma etiologia multifatorial. 
Não apenas a cultura tem o poder de influenciar no comportamento alimentar anormal. São necessários outros elementos para que as mulheres se tornem mais vulneráveis, como: a distorção e insatisfação com a imagem corporal e a autoestima, fatores altamente relacionados. A insatisfação corporal é fator de risco para o humor deprimido e para a baixa autoestima (Paxton, Neumark, Hannan \& Eisenberg, 2006; Wiseman, Peltzman, Halmi \& Sunday, 2004). A baixa autoestima e a insatisfação com a imagem corporal são fatores de risco para o surgimento de transtornos alimentares (Fairburn, Zafra, Boneca \& Welch, 1999).

Bruch (1973) identificou dois fatores importantes presentes nos transtornos alimentares: distorções na imagem corporal como causa mais próxima particularmente da anorexia nervosa e a dificuldade no desenvolvimento da identidade. O distúrbio da imagem corporal parece ser um dos mais graves sintomas nos transtornos alimentares; é mais complexo que a simples insatisfação corporal, tendo como consequência da distorção da imagem a insatisfação da mulher com seu corpo e a baixa autoestima. Esse fator, inclusive, foi incluído como um dos sintomas critérios para os transtornos anorexia e bulimia (APA, 2002).

Além da autoestima, insatisfação corporal e a distorção da imagem corporal, mulheres mais vulneráveis correm risco elevado de desenvolver algum tipo de transtorno alimentar. Outro desses fatores é a relação com a família, com a mãe, principalmente. Em geral, as teorias destacam a importância do contexto social imediato do indivíduo - particularmente a família - na determinação da autoestima.

\section{Relação mãe-filha e os transtornos alimentares}

O tema da relação entre mães e filhas sempre foi amplamente discutido. Bleichmar (1988) atribui que as mães tendem a vivenciar suas filhas mulheres como menos separadas delas, devido a componentes narcisistas que prevalecem nesta dupla como identificação e simbiose. Lerner (1990) ressalta que a tarefa de declaração da própria individualidade e diferença da mãe é relativamente mais difícil e complexa para a menina, porque é a filha, em particular, que pode experimentar inconscientemente que um passo para a autonomia é perigoso, como se estar separada e completa sem a mãe fosse uma traição desleal do relacionamento entre as duas.

É sabido que o desenvolvimento do psiquismo do sujeito se dá antes do seu nascimento (Bucaretchi, 2003) destaca que então, a fim de brecar a relação ilusória de plenitude mãe-bebê, a criança precisará lançar um olhar em direção à instância paterna, porque é o pai quem poderá equilibrar essa relação, afastando a criança do espaço materno onipotente. Mas, para que se possa produzir a interferência paterna, além do interesse da criança pelo pai, é essencial que a mãe também o aceite. Esta saída oferecida pelo pai, favorecida pela mãe e elaborada pela criança, vai constituir a estrutura necessária para a criança ser libertada como sujeito desejante.

No caso dos transtornos alimentares, ao invés de uma maternagem suficientemente boa, algo se inverteu no processo de interação entre mãe-filha, conforme ressalta Eliot (2004), já que insatisfações maternas e comportamentos instáveis são transmitidos aos filhos em geral, mesmo que silenciosamente.

Ward, Ramsay e Treasure (2000) identificaram que o vínculo mãe-filha é caracterizado por mais insegurança, medo de abandono e falta de autonomia nas mulheres com transtorno alimentar. Ward, Ramsay, Turnbull, Steele, Steele e Treasure. (2001) avaliaram o vínculo mãe e filha em vinte pacientes com anorexia nervosa, constatando que apenas uma possuía vínculo seguro. Latzer, Hochdorf, Bachar e Canetti (2002) e Tasca, Taylor, Ritchie e Balfour (2004) concluíram que os transtornos alimentares e suas atitudes devem ter relação com problemas de apego entre mãe e filha.

Grando e Rolim (2005) apontam que as mulheres com transtornos alimentares têm uma representação de sua família como um grupo social primário que faz cobranças e que, como elemento formador, tem participação na origem do distúrbio. Ainda a respeito dos modelos familiares Minuchin (1990) e SelviniPalazolli (1990) apontam um padrão que envolve, entre outros elementos, a dificuldade de separação e individuação entre os membros. Minuchin (1990) aponta que na família das anoréticas, existe a falta de um senso de identidade separada da matriz familiar e a incapacidade destas meninas de se separar de suas mães resultava numa falha em alcançar qualquer senso estável de seus próprios corpos, sentidos como se fossem habitados por um introjeto materno mau. Assim, a inanição poderia ser uma tentativa de interromper o crescimento desse objeto hostil e intrusivo. Nas famílias das bulímicas, o autor observou que os pais, com frequência se relacionavam com suas filhas como extensão de si próprios.

Ainda existem fatores observáveis no comportamento dos pais que influenciam na conduta da filha de forma mais direta, como um modelo. Dentre os estu- 
dos que comprovam essa afirmativa está o estudo de Agras, Hammer e McNicholas (1999) que, longitudinalmente, avaliaram recém-nascidos e seus pais no que diz respeito aos fatores preditivos de problemas alimentares na infância e constataram que a insatisfação corporal da mãe, a internalização do ideal de magreza, o comportamento de fazer dieta, os sintomas bulímicos e maior índice de massa corporal da mãe e do pai aumentam a chance de aparecimento de problemas alimentares na infância, o que pode aumentar o risco para transtornos alimentares no futuro. Outro estudo (Keery, Bouetelle, Van-den-Berg \& Thompon, 2005) examinou a prevalência e efeitos das críticas que os familiares fazem em relação à forma corporal das adolescentes, os resultados apontam um índice de $13 \%$ de críticas provindas da mãe e $19 \%$ do pai. As garotas que sofreram com comentários depreciativos apresentaram mais insatisfação corporal, baixa autoestima, mais comportamentos alimentares anormais e sintomas depressivos do que garotas não submetidas a essa situação.

Mães de pacientes com transtornos alimentares tendem a ser mais críticas e preocupadas com relação ao peso de suas filhas, incentivando-as a fazer dieta, mais do que as mães de filhas sem o transtorno (Gowers \& Shore, 2001). Ricciardelli e McCabe (2001) afirmam que a pressão para perder peso, exercida pela mãe, é o principal fator preditivo de insatisfação corporal e do engajamento em estratégias para modificar o corpo em adolescentes.

Um rígido controle e pais perfeccionistas limitam as oportunidades para o desenvolvimento de um self com funcionamento autônomo, prejudicando o desenvolvimento de um senso de self claro e elaborado (Bruch, 1973). A autora argumenta que a anorexia, dessa forma, acaba ocupando uma forma de controle, para a mulher demonstrar seu aparente senso de identidade, de competência e de eficiência. Stein e Corte (2003) revelam que como a criança sem uma identidade emergente encontra mudanças biológicas e sociais associadas a sua adolescência, essa falta de autonomia a submete a sentimentos de incompetência, dúvidas e medos de perda de controle, e para compensar isto, se volta ao peso corporal, um domínio culturalmente valorizado, como uma forma viável de definição do seu self. Os comportamentos que caracterizam a anorexia e a bulimia teriam a função de restaurar um senso de coesão, crescimento de sentimentos de eficiência e serve como uma "identidade compensatória" que permite alguma artificial presença significativa no mundo.
As origens de desenvolvimento das bulímicas são descritas por Goodsitt (1983) e Sugarman e Kurash (1982). De acordo com o primeiro, havia uma grande dificuldade de separação tanto nos pais quanto nas pacientes e que um tema comum na história do desenvolvimento delas era a ausência de um objeto transicional para ajudar a criança a separar-se psicologicamente de sua mãe. No outro estudo (Sugarman \& Kurash, 1982) os autores ponderaram que o esforço de desenvolvimento realizado pelas bulímicas para se separar de suas mães poderia, então, ser atuado pela utilização do próprio corpo como um objeto transicional, e assim, a ingestão de alimentos seria uma forma de desejo de fusão com a mãe, e a expulsão, seria um esforço para separar-se dela. Appledorn (2000) reforçou esse achado, concluindo que havia correlação significativa entre a gravidade da bulimia, a dificuldade nas relações objetais e os distúrbios de identidade.

\section{Autoestima, apego e os transtornos alimentares}

A autoestima é uma atitude positiva ou negativa voltada a um objeto particular: o si mesmo. Mas o conceito tem duas conotações muito diferentes. Uma conotação da autoestima implica o indivíduo pensar que é "muito bom"; outra muito diferente se relaciona com o crer ser: "suficientemente bom". É possível que uma pessoa se considere superior à maioria das demais, e então, se sinta inadequada, de acordo com certas regras que imponha a si mesmo. Inversamente, uma adolescente pode considerar-se um individuo comum, e apesar disso, estar satisfeita com o seu eu (Rosenberg, 1973). A construção de uma boa autoestima surge como alicerce de força de vida, está profundamente associada à resiliência, ou seja, à combinação entre flexibilidade e força para enfrentar os obstáculos, à criatividade para encontrar saídas, à visão otimista, à esperança, à fé e ao cultivo da alegria pelas coisas simples (Assis \& Avanci, 2004).

O apego é um vínculo afetivo, estável e consistente, que se estabelece entre mãe e bebê como resultado da interação entre ambos e envolve um sentimento de confiança básico. Também se caracteriza pela necessidade que sente o filho de buscar e manter certo grau de proximidade e de contato físico com as figuras com as quais tem vínculos (Bowlby, 1990). O modo como a criança constrói o conceito de si mesma, a partir das interações com seus pais, é de vital importância para seu futuro. O modelo que o filho configura será tanto mais seguro, vigoroso, estável e confiável quanto melhor apegado for à sua figura materna e quanto mais acessível, digna de confiança, disponível, esti- 
mulante e reforçadora tenha sido a conduta da mãe. Do contrário, o modelo que a criança tem de si mesma será inseguro, frágil, instável e desconfiado em função de como perceber a interação com seus pais, de forma mais hostil, desconfiada, distante ou inacessível (Polaino-Lorente, 2004).

Dependendo do tipo de apego existente entre mãe e filho (se inseguro, esquivo, ambivalente, desorganizado ou ansioso), a autoestima do filho terá nuances diferenciadas. Só o tipo de apego seguro, caracterizado pela percepção de que a mãe é uma base segura, disponível, responsiva e que em situações adversas é sensível, acessível e colaboradora, pode contribuir para que a autoestima da criança seja alicerçada numa forte segurança (Mruck, 1998).

A teoria do apego define que o modelo de relações primárias com os cuidadores influencia na cognição, no emocional e nas experiências comportamentais na vida do indivíduo (Bowlby, 1989). O autor hipotetizou que baseadas nos vínculos primários, as crianças constroem modelos de seu self e modelos dos outros. Rosenberg (1973) afirma que o indivíduo internaliza as ideias e atitudes expressas por figuras-chave de sua vida e pela cultura, passo importante para se apreender as bases da formação da autoestima. O autor ainda ressalta que a criança pequena não pode penetrar diretamente em sua própria experiência, pois primeiro percebe a forma como as pessoas reagem a ela, experimenta os próprios sentimentos e reações, para então aprender a pensar em si mesma.

A formação da adequada autoestima depende profundamente do olhar amoroso de apreciação, do ser visto como pessoa de valor, com competência, no mínimo por uma pessoa significativa nos círculos de convivência. $O$ papel de destaque nesse processo pertence à família, independentemente de sua composição, porque é a principal fonte de apoio estável (Assis \& Avanci, 2004).

Ramalho (2001) destaca que a imagem conferida pela mãe é adquirida a partir do olhar, do seu desejo, enquanto encarnação do outro primordial, tomado como espelho. Assim, nunca é com seus próprios olhos que a criança se vê, mas sempre com os olhos do outro. $\mathrm{O}$ ver-se numa identificação com esse olhar dirigido para si constitui, assim, o narcisismo. O que é, ainda, essencial na assunção da imagem do corpo no espelho é que a criança carregada pela mãe, cujo olhar a reconhece, se vira para ela como para the pedir que autentique, que testemunhe sua descoberta, trata-se do reconhecimento da sua mãe. Portanto, se a autoimagem, de natureza essencialmente narcísica, captada pela criança na aurora de sua vida psíquica for tíbia e fugidia, dará origem a um sentimento de integridade narcísica e de autoestima na mesma proporção tíbio e fugidio.

A estima que o filho sente pela mãe está ligada ao desenvolvimento de sua própria autoestima condicionada pela mãe (Polaino-Lorente, 2004). Mães de filhos com elevada autoestima tendem a ter relações mais positivas e a possuir maior equilíbrio, fornecendo respostas claras aos filhos e favorecendo sua independência. Mães de crianças de baixa autoestima tendem a ser emocionalmente instáveis (Coopersmith, 1967).

Quando o resultado de experiências arcaicas do desenvolvimento é desfavorável, há um aumento dos riscos para o desenvolvimento psíquico saudável, e um deles é a baixa autoestima e os transtornos alimentares (Gorgati, Holcberg \& Oliveira, 2002). A relação entre a baixa autoestima e os transtornos alimentares tem sido identificada em vários estudos e foi identificada como fator preditor de sintomas bulímicos por Vohs, Bardone, Joiner, Abramson e Heatherton (1999). Os autores investigaram a interação de fatores, como o perfeccionismo, percepção do peso e autoestima em 342 adolescentes para avaliar se eram preditores de sintomas bulímicos. Foi demonstrado que as adolescentes, que tinham altos índices de perfeccionismo e que se consideravam acima do peso, exibiam mais sintomas bulímicos, se a autoestima também era baixa; do contrário, as adolescentes, nas mesmas condições, mas que apresentavam alta autoestima, apresentavam menor sintomatologia. Os autores, Vohs, Pettit, Bardone, Katz, Abramson, Heatherton e Joiner (2001) replicaram o estudo, adicionando outras variáveis, mas no que se refere à autoestima e os dados foram novamente confirmados.

Abreu e Cangelli (2004) identificaram que as mulheres com bulimia nervosa apresentam uma autoestima flutuante, ou seja, quando têm a sensação de controle, sua autoestima é alta, quando se descontrolam, têm compulsões e purgações e sua autoestima se rebaixa. Mas Aranda, Murcia, Gil e Ruiloba (2001) compararam quatro grupos, um que apresentava sintomas anoréxicos, outro que apresentava sintomas bulímicos, e um grupo controle subdividido em dois, um com e outro sem comportamento restritivo. No grupo da anorexia nervosa (AN) e bulimia nervosa (BN) foram encontrados índices significativamente maiores de baixa autoestima e insatisfação com o próprio corpo do que no grupo controle sem restrição 
alimentar. Houve correlação positiva entre as variáveis sintomatológicas alimentares e a baixa autoestima, o que concorda com os vários estudos semelhantes que sugerem uma pobre autoestima e autoconceito nos transtornos alimentares, em maior medida do que o observado na população geral e grupos controles (Berrocal, Rivas, Barsabé \& Castro, 2003; Goldner, Geller, Birmingham \& Remick, 2000; Rosen \& Ramirez, 1998).

\section{Tratamento e prevenção: importância da família e da autoestima}

Apesar de os transtornos alimentares serem quadros bastante graves e deixarem lacunas na compreensão de sua etiologia, dificultando assim, um planejamento para prevenção primária e para os tratamentos, podem ser encontrados dados bastante positivos tanto no campo da prevenção quanto do tratamento.

Um dado otimista foi publicado por Halvorsen e Heyerdahl (2006) que ao investigarem a personalidade, a autoestima e a satisfação na vida em um grupo de anoréticas que haviam iniciado tratamento há, em média, oito anos, descobriram que as pacientes que haviam obtido sucesso no tratamento podiam apresentar traços normais de personalidade e de autoestima na idade adulta. Mas Pereira, Lock e Oggins (2006) enfatizam que é fundamental que a família também se desempenhe na formação de uma aliança com o tratamento de suas filhas com anorexia nervosa, uma vez que isso pode contribuir para o desfecho favorável do tratamento.

A autoestima é componente de peso na constituição desses transtornos e por isso se torna objetivo de tratamento manejar com ela para que se eleve a patamares mais seguros. As estratégias sugeridas para o tratamento da anorexia nervosa objetivam, entre outros focos, o aumento da autoestima porque, para as pacientes com transtornos alimentares, a magreza estaria associada à competência, superioridade e sucesso, tornando-se assim intrinsecamente associada à autoestima. A abordagem da autoestima envolve a redução das altas expectativas de desempenho das pacientes com anorexia, desenvolvendo padrões realistas de autoavaliação e incentivando-as a manter o foco em seus sucessos e qualidades. É importante também desenvolver uma avaliação multifacetada de valor pessoal, fazendo sua autoestima se apoiar em outros atributos além da aparência. Na bulimia nervosa, a modificação das crenças centrais e dos pensamentos associados à alimentação, aparência e valor pessoal, são realizados nos mesmos moldes que na anorexia (Duchesne \& Almeida, 2002).

A baixa autoestima está relacionada e presente nos mais variados transtornos psiquiátricos e por isso acaba sendo uma necessidade a ser resgatada ou desenvolvida na paciente. A baixa autoestima é sintoma presente nas ansiedades (Silva \& Costa, 2005), dependência química (Silva e Mattos, 2004), fator de risco para o uso de drogas (Feijó, 2001), distimia (Spanemberg \& Juruena, 2004), depressão (Bahls, 2002), esquizofrenia (Araújo, Petribú \& Bastos, 2002), transtorno de déficit de atenção (Souza, Serra, Mattos \& Franco, 2001) e comer noturno (Gluck, Geliebter \& Satov, 2001) e também se associa a sofrimentos em decorrência de abuso físico (Cecconello, Antoni \& Koller, 2003), violência contra mulher (Vianna, Bonfim \& Chicone, 2006) e malformações congênitas (Andrade \& Angerami, 2001). Além da violência, outras situações adversas à vida da criança e do adolescente costumam estar relacionadas a níveis mais baixos de autoestima: abuso de drogas, gravidez precoce, fracasso escolar, delinquência, ideias de morte, agressões escolares e depressão (Assis \& Avanci, 2004).

Intervenções desde a primeira infância, focadas nas relações parentais, e principalmente mãe-bebê, que as ensinem a identificar adequadamente e a responder às necessidades do bebê seriam importantes fatores para o desenvolvimento saudável do self, podendo dar suporte, consequentemente, ao futuro adolescente e adulto, vindo a servir de prevenção primária para o desenvolvimento de transtornos alimentares e várias outras psicopatologias, já que a autoestima é um importante fator presente na saúde mental (Mruck, 1998).

Em 1967, Coopersmith já salientava cinco condições que contribuem para melhorar a autoestima da criança: a) apresentar uma aceitação de seus pensamentos, sentimentos e valores pessoais; b) receber limites justos e definidos; c) os pais não serem autoritários, nem violentos para controlar e manipular a criança, nem humilhá-la ou ridicularizá-la; d) os pais devem manter altos padrões e altas expectativas em termos de comportamentos e desempenhos da criança; e) os pais devem apresentar um bom nível de autoestima, porque são modelos de aprendizagem. Crianças e jovens que se sentem amados, respeitados, escutados, compreendidos, valorizados e estimulados a desenvolverem suas habilidades e competências têm maior probabilidade de apresentar autoestima adequada. É inevitável constatar que esses critérios não foram res- 
peitados na história de vida das mulheres com transtornos alimentares.

\section{CONSIDERAÇÕES FINAIS}

A autoestima é tão importante para o ser humano que Maslow (1991) desenvolveu a ideia de que entre as necessidades humanas está a de estima, ou seja, a necessidade de autoestima e estima por parte dos outros. A satisfação dessa necessidade leva o indivíduo a se sentir confiante em si mesmo (no seu valor, força, capacidade e adequação), mais útil e necessário ao mundo.

As adolescentes com elevada autoestima tendem a um "afeto positivo", acreditam na sua competência e no seu valor, demonstram capacidade para lidar com desafios, são autossuficientes e conseguem se adaptar mais facilmente a uma situação nova. Essas pessoas são independentes, autônomas e percebem a realidade mais acuradamente. Já, as adolescentes com baixa autoestima são mais sensíveis a críticas dirigidas a elas, sofrem de um "afeto negativo", com sentimentos de inferioridade, menos-valia, isolamento e insegurança e têm um grande senso de proteção consigo; a vulnerabilidade é uma característica marcante. Por meio da literatura é possível ponderar que a baixa ou alta autoestima determina o curso que a pessoa pode dar à sua vida, porque afeta suas decisões e o seu estado emocional.

Para estimar-se, é preciso, fundamentalmente, que um outro tenha nos estimado, favorecendo a crença de que somos dignos de amor e de estima. Por isso, pensamos logo na figura primordial: a figura materna. Embora existam tentativas de prevenção, de técnicas para tratamento, há um fator que não pode ser ignorado: a família, principalmente a mãe. Por esse motivo deve ser lançado um olhar especial para esta figura, pelo poder que exerce como modelo no comportamento da criança, da adolescente e consequentemente da futura mulher adulta.

\section{REFERÊNCIAS}

Abreu, C. N., \& Cangelli, R. (2004). Anorexia nervosa and bulimia nervosa: A psychotherapeutic cognitive-constructivist approach. Revista de Psiquiatria Clínica, 31(4), 177-183.

Agras, W. S. (2001). The consequences and costs of the eating disorders. The Psychiatric Clinics of North America, 24, 371379.

Agras, S., Hammer, L., \& McNicholas, F. (1999). A prospective study of the influence of eating-disordered mothers on their children. International Journal of Eating Disorders, 25(3), 253-262.
American Psychiatric Association (2002). Manual diagnóstico e estatístico de transtornos mentais - DSM-IV-TR (4 ed.) (D. Batista, Trad.). Porto Alegre: Artmed.

American Psychiatric Association (2006). Practice guideline for the treatment of patients with eating disorders $\left(3^{\mathrm{a}} \mathrm{ed}.\right)$. Washington, DC: American Psychiatric Association.

Andrade, D., \& Angerami, E. L. S. (2001). The self-esteem of adolescents with and without a cleft lip and/or palate. Revista Latino-Americana de Enfermagem, 9(6), 37-41.

Appledorn, K. (2000). Object relations and identity disturbances in bulimic women. Dissertation Abstract International, 60(12B), 6394 .

Araújo, F. S, Petribu, K., \& Bastos, O. (2002). Depressão na esquizofrenia. Revista Brasileira de Psiquiatria, 24, 86-93.

Assis, S. G., \& Avanci, J. Q. (2004). Labirinto de espelhos: formação da autoestima na infância e na adolescência. Rio de Janeiro: Fiocruz.

Assumpção, C. L., \& Cabral, M. D. (2002). Complicações clínicas da anorexia nervosa e bulimia nervosa. Revista Brasileira de Psiquiatria, 24, 29-33.

Bahls, S. C. (2002). Aspectos clínicos da depressão em crianças e adolescentes. Jornal de Pediatria, 78(5), 359-366.

Bardone, A. M., \& Cass, K. M. (2006). Investigating the impact os pro-anorexia websites: A pilot study. European Eating Disorders Rewiew, 14(4), 256-265.

Binford, R. B., \& Le Grange, D. (2005). Adolescents with bulimia and eating disorder not otherwise specified purging only. International Journal of Eating Disorder, 38(2), 157-161.

Bleichmar, E. (1988). O feminismo espontâneo da histeria. Porto Alegre: Artes Médicas.

Bowlby, J. (1989). Uma base segura: Aplicações clínicas da teoria do apego. Porto Alegre: Artes Médicas.

Bowlby, J. (1990). Apego e perda: Apego, a natureza do vínculo ( $2^{\mathrm{a}}$ ed.). São Paulo: Martins Fontes.

Berrocal, C., Rivas, T., Barsabé, R., \& Castro, S. (2003). Análisis de las diferencias clínicas entre la bulimia nerviosa y la bulimia por debajo del umbral. Psicología Conductual, 11(2), 239-259.

Bucaretchi, H. A. (2003). Anorexia e bulimia nervosa: Uma visão multidisciplinar. São Paulo: Casa do Psicólogo.

Bruch, H. (1973). Eating disorders - Obesity, anorexia nervosa and the person within. New York: Basic Books, Harper Torchbooks.

Cameron, E. M., \& Ferraro, F. R. (2004). Body satisfaction in college women after brief exposure to magazine images. Perceptual and Motor Skills, 98(3), 1093-1099.

Cecconello, A. M., De Antoni, C., \& Koller, S. H. (2003). Práticas educativas, estilos parentais e abuso físico no contexto familiar. Psicologia em Estudo, 8, 45-54.

Coopersmith, S. (1967). The antecedents of self-esteem. San Francisco: Freeman.

Cordás, T. A. (2004). Transtornos alimentares: Classificação e diagnóstico. Revista de Psiquiatria Clínica, 31(4), 154-157.

Cordás, T. A., \& Claudino, A. M. (2002). Transtornos alimentares: Fundamentos históricos. Revista. Brasileira de Psiquiatria, 24(3), 3-6. 
Duchesne, M., \& Almeida, P. E. M. (2002). Terapia cognitivocomportamental dos transtornos alimentares. Revista Brasileira de Psiquiatria, 24, 49-53.

Eliot, A. O. (2004, Spring). A concept of self in eating-disordered adolescent girls: A consideration of genetic factors. Annals of the American Psychoterapy Association. Reitrado em 2 de junho de 2008, de http://findarticles.com/p/articles/mi hb013/is 1_7/ai_n29084498/.

Fairburn, C. G., Zafra, C., Boneca, H. A., \& Welch, S. L. (1999) Risk factors for anorexia nervosa: Three integrated casecontrol comparisons. Archives of General Psychiatry, 56, 468476.

Feijó, R. B., \& Oliveira, E. A. (2001). Comportamento de risco na adolescência. Jornal de Pediatria, 77(2), 125-134.

Filippi, L. M. (2004). Eating disorders as biologiclly and culturally determined mental illnesses. Scan's Pulse, 23(3), 9-12.

Gluck, M. E., Geliebter, A., \& Satov, T. (2001). Night eating syndrome is associated with depression, low self-esteem, reduced daytime hunger, and less weight loss in obese outpatients. Obesity Research, 9, 264-267.

Goldner, E. M., Geller, J., Birmingham, L., \& Remick, R. (2000). Comparison of shoplifting behaviours in patients with eating disorders, psychiatric control subjects, and undergraduate control subjects. Canadian Journal of Psychiatry, 45(5), 471-475.

Goodsitt, A. (1983). Self-regulatory disturbances in eating disorders. International Journal of Eating Disorders, 2(3), 51-60.

Gorgati, S. B., Holcberg, A., \& Oliveira, M. D. (2002). Psychodynamic approach on treatment of eating disorders. Revista. Brasileira de Psiquiatria, 24, 44-48.

Gowers, S. G., \& Shore, A. (2001). Development of weight and shape concerns in the aetiology of eating disorders. British Journal of Psychiatry, 179, 236-242.

Grando, L. H., \& Rolim, M. A. (2005). Família e transtornos alimentares: As representações dos profissionais de enfermagem de uma instituição universitária de atenção à saúde mental. Revista Latino-americana de Enfermagem, 13(6), 989-995.

Halvorsen, I., \& Heyerdahl, S. (2006). Girls with anorexia nervosa as young adults: Personality, self-esteem, and life satisfaction. International Journal of Eating Disorders, 39(4), 285-293.

Inchi, R., \& Meral, N. (2006). A content analysis of popular magazine articles on eating disorders. Eating Disorders, 14(2), 109-121.

Keery, H., Bouetelle, K., Van-den-Berg, P., \& Thompon, J. K. (2005). The impact of appearence-related teasing by family members. Journal of Adolescent Health, 7(2), 120-127.

Latzer, Y., Hochdorf, Z., Bachar, B., \& Canetti, L. (2002). Attachment style and family functioning as discriminating factors in eating disorders. Contemporary Family Therapy, 24(4), 581599.

Lerner, H. (1990). Mulheres em terapia. Porto Alegre: Artes Médicas.

Mas, M. V., Aranda, F., Murcia, S., Gil, T., \& Ruiloba, J. (2001). Baja autoestima, restricción alimentaria y variables psicopatológicas en anorexia y bulimia nerviosa: Un estudio de casos controles. Psicología Conductual, 9(2), 267-278.
Maslow, A. H. (1991). Motivacion y personalidad. Madrid: Diaz de Santos.

Minuchin, S. (1990). Famílias: Funcionamento e tratamento. Porto Alegre: Artmed.

Monro, F. J., \& Huon, G. F. (2006). Medria-protreyed idealized images, self-objectification and eating behavior. Eating Behaviors, 7, 375-383.

Morgan, C. M., Vecchiatti, I. R., \& Negrão, A. Z. (2002). Etiologia dos transtornos alimentares: Aspectos biológicos, psicológicos e sócio-culturais. Revista Brasileira de Psiquiatria, 24, $18-23$.

Mruck, C. (1998). Autoestima: Investigación, teoría y práctica. Bilbao: Desclée de Brouwer.

Paxton, S. J., Neumark, S., Hannan, P. J., \& Eisenberg, M. E. (2006). Body dissatisfaction prospectively predicts depressive mood and low self-esteem in adolescent girls and boys. Journal of Clinical Child and Adolescent Psychology, 35(4), 539-549.

Pereira, T., Lock, J., \& Oggins, J. (2006). Role of therapeutic alliance in family therapy for adolescent anorexia nervosa. The International Journal of Eating Disorders, 39(8), 677-684

Pillon, S. C., \& Luis, M. A. V. (2004). Explanatory models for alcohol and drugs use and the nursing practice. Revista LatinoAmericana de Enfermagem, 12(4), 676-682.

Pinzon, V., \& Nogueira, F. C. (2004). Epidemiologia, curso e evolução dos transtornos alimentares. Revista de Psiquiatria Clínica, 31(4), 158-160.

Polaino-Lorente, A. (2004). Familia y autoestima. Barcelona: Ariel.

Ramalho, R. M. (2001). Anorexia e bulimia: Manifestações do sofrimento feminino hoje. Dissertação de Mestrado nãopublicada. Pontifícia Universidade de São Paulo.

Ricciardelli, L. A., \& McCabe, M. P. (2001). Children's body image concerns and eating disturbance: A review of the literature. Clinical Psychology Review, 21(3), 325-344.

Rosen, J. C., \& Ramirez, E. (1998). A comparison of eating disorders and body dysmorphic disorder on body image and image psychological adjustment. Journal of Psychossomatic Research, $44,441-449$

Rosenberg, M. (1973). La autoimagen del adolescente y la sociedad. Buenos Aires: Paidós.

Russell, G. F. M. (1979). Bulimia nervosa: An ominous variant of anorexia nervosa. Psychological Medicine, 9, 429-448.

Segatto, C., Padilla, I., \& Frutuoso, S. (2006, 18 de novembro). Por dentro da mente de uma anoréxica. Época, p. 55.

Selvini-Palazolli, M. (1990). Los juegos psicóticos en la familia. Buenos Aires: Paidós.

Silva, M., \& Costa, M. (2005). Desenvolvimento psicossocial e ansiedades nos jovens. Análise Psicológica, 2(23), 111-127.

Silva, V. A., \& Mattos, H. F. (2004). Os jovens são mais vulneráveis as drogas?. Em Pinsky, I. \& Bessa, M. A. (Orgs.). Adolescência e drogas (pp. 31-44). São Paulo: Contexto.

Souza, I., Serra, M. A., Mattos, P., \& Franco, V. (2001). Comorbidades em crianças e adolescentes com transtorno do déficit de atenção: Resultados preliminares. Arquivos de Neuropsiquiatria, 59(2-B), 401-406. 
Spanemberg, L., \& Juruena, M F, (2004). Dysthymia: Historical/nosological characteristics and its relationship with major depressive disorder. Revista de Psiquiatria do Rio Grande do Sul, 26(3), 300-311.

Stein, K. F., \& Corte, C. (2003) Reconceptualizing causative factors and intervention strategies in the eating disorders: A shift from body image to self-concept impairments. Archives of Psychiatric Nursing, 17(2), 57-66.

Sugarmam, A., \& Kurash, C. (1982). The body as a transicional object in bulimia. International Journal of Eating Disorders, 1(4), 57-67.

Tasca, G. A., Taylor, D., Ritchie, K., \& Balfour, L. (2004). Attachment predicts treatment completion in an eating disorders partial hospital program among women with anorexia nervosa. Journal of Personality Assessment, 83(3), 201-212.

Ward, A., Ramsay, R., \& Treasure, J. (2000). Attachment research in eating disorders. British Journal of Medical Psychology, 73, $35-51$.

Ward, A., Ramsay, R., Turnbull, S., Steele, M., Steele, H., \& Treasure J. (2001). Attachment in anorexia nervosa: A transgenerational perspective. British Journal of Medical Psycho$\log y, 74(4), 497-505$.
Wiseman, C. V., Peltzman, B., Halmi, K. A., \& Sunday, S. R. (2004). Risk factor for eating disorders: Surprising similarities between middle school boys and girls. Eating Disorders, 12(4), 315-320.

Vianna, L., Bomfim, G., \& Chicone, G. (2006). Autoestima de las mujeres que sufrieron violências. Revista Latino-Americana de Enfermagem, 14(5), 695-701.

Vohs, K., Bardone, A., Joiner, T., Abramson, L. Y., \& Heatherton, T. (1999). Perfectionism, perceived weight status, and selfesteem interact to predict bulimic symptoms: A model of bulimic symptom development. Journal of Abnormal Psychology, 188(4), 695-700.

Vohs, K., Pettit, Z., Bardone, A., Katz, J., Abramson, L., Heatherton, T., \& Joiner, T. (2001). Perfectionism, body dissatisfaction and self-esteem: An interactive model of bulimic symptom development. Journal of Social and Clinical Psychology, 20(4), 476-497.

Recebido: 05/05/2007

Última revisão: 05/10/2008 Aceite final: 10/11/2008

\section{Nota:}

${ }^{1}$ Esse artigo é resultante da Dissertação de Mestrado da primeira autora, sob orientação do segundo, com o apoio institucional da CAPES e CNPq.

\section{Sobre os autores:}

Daniela Sopezki: Psicóloga e Pedagoga. Especialista em Psicoterapias Cognitivo-Comportamentais. Mestra em Psicologia Clínica pela Pontifícia Universidade Católica do Rio Grande do Sul (PUCRS).

Cícero E. Vaz: Psicólogo, Professor e Pesquisador da Pontifícia Universidade Católica do Rio Grande do Sul (PUCRS)

Endereço para correspondência: Rua Baronesa do Gravataí, 152, ap. 34 - Bairro: Cidade Baixa - 90160-070 Porto Alegre - RS.

Endereço eletrônico: danielasopezki@ hotmail.com. 